NOTICE: This is the author's version of a work that was accepted for publication by Elsevier. Changes resulting from the publishing process, such as peer review, editing, corrections, structural formatting, and other quality control mechanisms may not be reflected in this document. Changes may have been made to this work since it was submitted for publication. A definitive version was subsequently published by Elsevier.

(C) 2017. This manuscript version is made available under the CC-BY-NC-ND 4.0 license http:// creativecommons.org/licenses/by-nc-nd/4.0/ 


\section{Taking laser Doppler vibrometry off the tripod: correction of measurements affected by instrument vibration}

Ben J. Halkon, Steve J. Rothberg

Wolfson School. Mechanical, Electrical and Manufacturing Engineering

Loughborough University, Loughborough, Leicestershire, LE11 3TU, UK.

Corresponding author: Ben Halkon

e-mail: b.j.halkon@lboro.ac.uk,

tel: +44 (0) 1509564823 


\title{
Taking laser Doppler vibrometry off the tripod: correction of measurements affected by instrument vibration
}

\author{
Ben J. Halkon, Steve J. Rothberg \\ Wolfson School. Mechanical, Electrical and Manufacturing Engineering \\ Loughborough University, Loughborough, Leicestershire, LE11 3TU, UK.
}

\begin{abstract}
Laser Doppler vibrometers (LDVs) are now well-established as an effective non-contact alternative to traditional contacting transducers. Despite 30 years of successful applications, however, very little attention has been given to sensitivity to vibration of the instrument itself. In this paper, the sensitivity to instrument vibration is confirmed before development theoretically and experimentally of a practical scheme to enable correction of measurements for arbitrary instrument vibration. The scheme requires a pair of correction sensors with appropriate orientation and relative location, while using frequency domain processing to accommodate inter-channel time delay and signal integrations. Error reductions in excess of $30 \mathrm{~dB}$ are delivered in laboratory tests with simultaneous instrument and target vibration over a broad frequency range. Ultimately, application to measurement on a vehicle simulator experiencing high levels of vibration demonstrates the practical nature of the correction technique and its robustness in a challenging measurement environment.
\end{abstract}

KEYWORDS: laser Doppler vibrometry; non-contact surface vibration measurement; correction for instrument vibration; measurement uncertainty. 


\section{Introduction}

The laser Doppler vibrometer (LDV) is now well-established as an effective non-contact alternative to traditional contacting transducers such as the ubiquitous piezoelectric accelerometer. LDVs measure vibration velocity and are technically well-suited to general application but they have repeatedly proved invaluable in a variety of challenging measurement scenarios. Commercial LDVs have now been available for more than 30 years but, despite so much successful application, almost no attention has been given to a quite fundamental aspect of LDV operation which is that the measurement is of velocity relative to the instrument itself. Consequently, measurements are directly affected by instrument vibration in the direction of the laser beam and this cannot be distinguished from the intended measurement.

While instrument vibrations can be a factor in general application, they are particularly important in specific scenarios including handheld measurements such as in a clinical application [1], measurements taken within a moving structure such as a vehicle cabin [2], and measurements taken from a moving vehicle [3]4]. To date, the routine approach taken is to attempt to isolate the instrument to minimise its vibration, often by mounting on a tripod with compliant feet, but this is not always convenient or adequate. This paper shows for the first time how to compensate for instrument vibrations, contributing significantly to the advancement of LDV as a user-friendly technique. This comprehensive study incudes a confirmation of the measurement sensitivity to such motions, a theoretical basis for the proposed scheme of additional measurements for correction in the presence of complex instrument motions, and laboratory tests to confirm its effectiveness. Finally, the system is taken out of the laboratory and successfully implemented on a flight simulator platform undergoing extreme vibrations.

\section{Understanding the velocity measured during instrument vibration}

\section{Extent of the problem}

The sensitivity to instrument vibration in the direction of the laser beam was confirmed by a simple experiment, as outlined in Figure 1, in which two simultaneous LDV measurements were made on a (nominally) stationary target. The "Vibrating" LDV (Polytec OFV4000 set-up for translational vibration measurement) is mounted on a shaker and excited; the excitation is broadband and primarily in the direction of the laser beam. A "Fixed" LDV (Polytec PDV100) (i.e. not excited) is tripod mounted and used to provide the 'true' measurement. As shown in Figure 2, the true measurement is at an extremely low level, as expected, but the measurement from the vibrating instrument shows significant sensitivity to instrument vibration in the direction of the laser beam, as suggested in Section 1.

[INSERT: Figure 1. Experimental arrangement showing vibrating and fixed LDVs to demonstrate sensitivity to instrument vibration; a) schematic and b) physical set-up] 
[INSERT: Figure 2. Comparison between measurements from the vibrating LDV and from the fixed LDV on a (nominally) stationary target]

The fundamental requirement for correction is an independent measurement of the instrument velocity in the direction the laser beam at some point along the laser beam path. However, such a measurement is impractical because it would obscure the laser beam so one or more compromise locations have to be chosen where one or more absolute motion sensors can be attached. This proposal relies on the reasonable assumption that the region of the optical head of the vibrometer including the laser beam aperture and any sensor locations moves as a rigid body for the frequency range of interest. In the first stage of this investigation, an accelerometer was attached to the front face of optical head and its output integrated to velocity for easy comparison, which is shown in Figure 3.

[INSERT: Figure 3. For a nominally stationary target, comparison between measurements from the vibrating LDV and an accelerometer mounted on the vibrometer optical head body to one side of the beam emitter tube; instrument vibration $160 \times 10^{-3} \mathrm{~g}$ RMS]

Figure 3 illustrates two important issues. Firstly, the visual comparison between the two measurement spectra is reasonable overall. This supports the observation that the LDV measurement sensitivity to the instrument's own motion in the direction of the laser beam is $100 \%$. At the same time, the comparison is far from perfect because of the compromise required in locating the accelerometer. The mean of the absolute percentage differences at individual spectral lines is still $100.4 \%$ from $2.5-100$ Hz. Given that, as shown in Figure 2, the apparent target velocity is two orders of magnitude higher than the true velocity in these tests, the single correction measurement already offers a valuable improvement in measurement accuracy and similar compensations have been reported in literature previously [5], [6]. However, the remaining error is far too large to present as a satisfactory outcome and, for this reason, this paper shows for the first time how an ideal means of determining the required instrument motion can be found to provide accurate and practical correction that is effective for arbitrary, six degree-of-freedom instrument vibration.

\section{Mathematical determination of the required correction}

Using a vector-based approach and with reference to Figure 4, the arbitrary velocity at the location of Accelerometer $1, \overrightarrow{V_{1}}$ can be written in terms of the arbitrary velocity at some chosen point, $O$, along the laser beam path, $\overrightarrow{V_{0}}$, and the arbitrary angular motion around the chosen point, $\vec{\theta}$ :

$$
\overrightarrow{V_{1}}=\overrightarrow{V_{0}}+\overrightarrow{r_{1}} \times \vec{\theta}
$$

where $\overrightarrow{r_{1}}$ is the position vector for the accelerometer location relative to the chosen point along the laser beam path.

[INSERT: Figure 4. Schematic showing required location of accelerometers to enable effective correction for instrument vibration in the direction of the laser beam] 
The required correction velocity, $U_{0}$, is the component of the total velocity in the laser beam direction, defined by the unit vector $\hat{x}$, and can be written as:

$$
U_{0}=\hat{x} \cdot \overrightarrow{V_{0}}
$$

From equation (1):

$$
\hat{x} \cdot \overrightarrow{V_{0}}=\hat{x} \cdot \overrightarrow{V_{1}}-\hat{x} \cdot\left(\overrightarrow{r_{1}} \times \vec{\theta}\right)
$$

$U_{1}=\hat{x} \cdot \overrightarrow{V_{1}}$ is the measurement made by Accelerometer 1 (after integration), while $\hat{x} \cdot\left(\overrightarrow{r_{1}} \times \vec{\theta}\right)$, in which $\vec{\theta}$ is currently unknown, is responsible for the difference apparent in Figure 3. Additional measurements of the instrument vibration are clearly required but the question is: how many measurements and at which location(s)? If an additional measurement, $U_{2}$, is made at a location defined by position vector $\overrightarrow{r_{2}}$ then:

$$
\overrightarrow{V_{2}}=\overrightarrow{V_{0}}+\overrightarrow{r_{2}} \times \vec{\theta}
$$

where $\overrightarrow{V_{2}}$ is related to this measurement as $U_{2}=\hat{x} \cdot \overrightarrow{V_{2}}$. An improved correction measurement can then be proposed in which these two measurements are summed and halved:

$$
0.5\left(\hat{x} \cdot \overrightarrow{V_{1}}+\hat{x} \cdot \overrightarrow{V_{2}}\right)=U_{0}+0.5\left(\hat{x} \cdot\left(\overrightarrow{r_{1}} \times \vec{\theta}\right)+\hat{x} \cdot\left(\overrightarrow{r_{2}} \times \vec{\theta}\right)\right)
$$

Accurate correction is achieved when $0.5\left(\hat{x} \cdot \overrightarrow{V_{1}}+\hat{x} \cdot \overrightarrow{V_{2}}\right)=U_{0}$ and therefore requires that the accelerometers are positioned such that the term containing the cross-products equates to zero. A simple rearrangement of that term shows how this can be achieved practically:

$$
\hat{x} \cdot\left(\overrightarrow{r_{1}} \times \vec{\theta}\right)+\hat{x} \cdot\left(\overrightarrow{r_{2}} \times \vec{\theta}\right)=\left(\overrightarrow{r_{1}}+\overrightarrow{r_{2}}\right) \cdot(\vec{\theta} \times \hat{x})
$$

If $\left(\overrightarrow{r_{1}}+\overrightarrow{r_{2}}\right)$ can be made zero then the aim is achieved. Indeed, the requirement is even less restrictive than this since the cross product $(\vec{\theta} \times \hat{x})$ includes only $\hat{y}$ and $\hat{z}$ components such that the vector sum $\left(\overrightarrow{r_{1}}+\overrightarrow{r_{2}}\right)$ only needs to have zero $\hat{y}$ and $\hat{z}$ components for the whole term to be zero. In practice this means that a single-axis accelerometer mounted on the instrument at any convenient location (and with its sensitive axis oriented in the same direction as the laser beam) must be paired with a second accelerometer with the same orientation and equal but opposite $(y, z)$ location coordinates. The accelerometers need not be located in the same $(y, z)$ plane, since matching $x$ location coordinates is not required for $\left(\overrightarrow{r_{1}}+\overrightarrow{r_{2}}\right) \cdot(\vec{\theta} \times \hat{x})$ to equate to zero, but mounting both accelerometers in the same $(y$, z) plane seems likely to be a generally convenient arrangement. In this study, the accelerometers were mounted on the front face of the instrument's optical head, as indicated in Figure 4.

Equations (4) and (5) can be used as the basis of an evaluation of the errors associated with imperfect positioning of the correction accelerometers. For example, an error $\Delta$ in the relative z-positions of the 
accelerometers, i.e. $\left(\overrightarrow{r_{1}}+\overrightarrow{r_{2}}\right)=\Delta \hat{z}$, in the presence of an angular vibration $\vec{\theta}=\theta_{y} \hat{y}$, will result in a measured velocity written as:

$$
U_{0}=\hat{x} \cdot \overrightarrow{V_{0}}+0.5 \Delta \hat{z} \cdot\left(\theta_{y} \hat{y} \times \hat{x}\right)=\hat{x} \cdot \overrightarrow{V_{0}}-0.5 \Delta \theta_{y}
$$

The associated error in the correction velocity is $\frac{\pi}{360} \mathrm{~mm} / \mathrm{s}$ per $\mathrm{mm}$ position error per degree angular vibration.

Another important error would be a small misalignment of an accelerometer by an angle $\delta$.

Neglecting angular vibrations, the sum of the accelerometer outputs would be:

$$
U_{0}=0.5\left(\sin \delta\left(\hat{y} \cdot \overrightarrow{V_{0}}\right)+\cos \delta\left(\hat{x} \cdot \overrightarrow{V_{0}}\right)+\hat{x} \cdot \overrightarrow{V_{0}}\right) \approx 0.5 \delta\left(\hat{y} \cdot \overrightarrow{V_{0}}\right)+\left(\hat{x} \cdot \overrightarrow{V_{0}}\right)
$$

The larger error in $U_{0}$ is then $0.5 \delta\left(\hat{y} \cdot \overrightarrow{V_{0}}\right)$. In the case of equal $x$ - and $y$-vibration components, this equates to a percentage error of $\frac{\pi}{3.6}$ or approximately $1 \%$ per degree of misalignment.

\section{Experimental confirmation of the required correction}

The effectiveness of this approach is confirmed experimentally in Figure 5. Prior to making these measurements, the frame on which the instrument optical head was mounted and fixed to the shaker was stiffened and the assembly mass better distributed about the shaker axis. The motivation for this was to flatten the response spectrum encountered in earlier measurements (for example, in Figure 3) to aid demonstration of the effectiveness of the correction technique. The modifications were not a requirement of the correction method which would work reliably in either case. These modifications had the secondary effect of reducing the very angular motions that equation $(2 b)$ has shown to be problematic for a single correcting measurement. Nonetheless, the combination of two correction measurements still brings a significant improvement.

Figure 5a shows the LDV measurement (stationary target, instrument vibration at $160 \times 10^{-3} \mathrm{~g}$ RMS broadband) and the corresponding correction measurement obtained with a single accelerometer. As a consequence of the modifications described above, the comparison is now closer to start with than in Figure 3 because of reduced angular motion but there remain regions of the spectrum, particularly towards the higher frequencies, where the agreement is insufficient for accurate correction. The mean of the absolute percentage differences at individual spectral lines is $7.8 \%$ from $2.5-100 \mathrm{~Hz}$, rising to $11.6 \%$ in the range from $50-100 \mathrm{~Hz}$. Introduction of the second accelerometer and addition of the two measured velocities, according to equation (4), improves still further the correction that can be made as can be seen in Figure 5b. The true measurement and the corresponding correction measurement are now almost indistinguishable visually. The mean of the absolute percentage differences at individual lines in the spectrum has reduced to $3.2 \%$ from $2.5-100 \mathrm{~Hz}$, and to $4.6 \%$ in the range from $50-100 \mathrm{~Hz}$. The result is very significant because it confirms that an optimum correction for arbitrary, six degree-of-freedom vibration of the instrument requires only two 
measurements in the laser beam direction provided their positions relative to each other have equal and opposite $(y, z)$ coordinates. This is an effective, simple and very practical solution to an important problem. The remaining error is comparable with that suggested by the simple sensitivity analysis based on equations $(6 a \& b)$.

[INSERT: Figure 5. Comparison between LDV measurement (stationary target, instrument vibration at $160 \times 10^{-3} \mathrm{~g}$ RMS broadband) and alternative correction measurements: a) single accelerometer b) optimum combination of two accelerometers]

The corrected measurement, $U_{\text {corr }}$, is now given by:

$$
U_{\text {corr }}=U_{m}-U_{0}
$$

where $U_{m}$ is the original LDV measurement and $U_{0}$ is the correction measurement from equation (4). In practice, attention must be paid to the positive sense for the LDV and accelerometers. In equation (7), all have a positive sense in the $+\hat{x}$ direction.

Secondary effects

Instrument vibration can cause perceptible motion of the laser beam on the target surface if displacement amplitudes are great enough. Successful measurements clearly rely on the laser beam remaining acceptably on the region of interest on the target. Even when this is the case, however, transverse sensitivities can have an effect on the accuracy of the corrected measurement. In LDV, pseudo-vibration (including speckle noise) [7] is associated with ultimate motion of the laser beam in $\hat{y}$ and $\hat{z}$ directions across the target surface. These can be a consequence of translational and/or angular motions of the instrument and they cannot be corrected by means proposed in this paper. In fact, they cannot be corrected by any known means. However, the sensitivity to these motions is of the order of $0.1 \%$ or less (for transverse motion) [7] and so the issue addressed in this paper, where sensitivity is $100 \%$, is on an altogether different scale. Accelerometers also have a transverse sensitivity, typically a few percent, which might degrade the quality of the correction depending on the relative motion amplitudes.

\section{Experimental investigations}

\section{Experimental arrangement-laboratory-based scenarios}

The experimental arrangement in Figure 6 shows the means by which the LDV vibration and target vibration are isolated and independently controllable. The target motion is generated by a small electrodynamic shaker (sat on the floor in the lower left hand side of the image in Figure 6b) placed with the axis of motion in the vertical direction. The vibrating LDV used is again a Polytec OFV 4000 set up for translational vibration measurement. As can also be seen in Figure $6 \mathrm{~b}$ (mid left hand side of the image), the OFV4000 optical head is mounted to a larger electrodynamic shaker so as to enable instrument vibration independently of target vibration and simultaneously. The fixed LDV, a Polytec 
PDV100, is positioned above the vibrating LDV (top left of the image) to provide a 'true' measurement. A small angle between the two laser beams enabled the incidence points to be in sufficiently close proximity on the target while allowing the PDV100 beam to avoid the OFV4000 optical head; a $5^{\circ}$ angle introduces an error of less than $0.5 \%$. The two accelerometers (identified in Figure 6a but not visible in the image in Figure 6b) were simply mounted on the front face of the optical head, equidistant about the beam and with a sensitive axis parallel to the beam as previously described and set out in Figure 4. They were signal conditioned using a Brüel \& Kjær Nexus Type 2692 charge amplifier. Finally, an industry standard LMS Test.Lab/SCADAS Mobile data acquisition system was used to acquire the various measured signals and to generate the necessary shaker signals for amplification.

[INSERT: Figure 6. Equipment arrangement for laboratory-based experimental investigation:

a) schematic and b) physical set-up]

The motion of the vibrating LDV is primarily in the direction of the laser beam (the shaker has a single axis of vibration) but no special measures were taken to reduce angular or other translational motions as the correction method works effectively for arbitrary motion as previously described. For example, the mount held the LDV on a cantilever to clear the side of the (large) shaker body and some angular motion resulted from this. Uncorrelated broadband vibrations were driven by both shakers to emphasise the versatility of the method, with a range of levels chosen to be representative of what might be encountered in real-world applications.

\section{Frequency domain processing}

The correction described in the section 2 can be made in either the time or the frequency domain. The use of accelerometers and the concomitant need to integrate outputs led to a decision to work in the frequency domain in this study and the means of processing is set out in this section. Working with the complex form for the $n$th frequency, $\omega_{n}$, component, the accelerometer outputs can be written as:

$$
\begin{aligned}
& {\left[a_{1}\right]_{\omega_{n}}=A_{1}\left(\omega_{n}\right) \exp \left[j\left(\omega_{n} t+\phi_{1}\left(\omega_{n}\right)\right)\right]} \\
& {\left[a_{2}\right]_{\omega_{n}}=A_{2}\left(\omega_{n}\right) \exp \left[j\left(\omega_{n} t+\phi_{2}\left(\omega_{n}\right)\right)\right]}
\end{aligned}
$$

in which $A_{1}$ and $A_{2}$ are the amplitudes and $\phi_{1}$ and $\phi_{2}$ are the phases of each frequency component in the Fourier transform. The Fourier transforms can be written as follows (with $\left(\omega_{n}\right)$ omitted for brevity):

$$
\begin{aligned}
& {\left[F T\left(a_{1}\right)\right]_{\omega_{n}}=A_{1} \cos \phi_{1}+j A_{1} \sin \phi_{1}} \\
& {\left[F T\left(a_{2}\right)\right]_{\omega_{n}}=A_{2} \cos \phi_{2}+j A_{2} \sin \phi_{2}}
\end{aligned}
$$

Integration in the frequency domain provides the following velocities: 


$$
\begin{aligned}
& {\left[F T\left(U_{1}\right)\right]_{\omega_{n}}=\frac{1}{\omega_{n}}\left(A_{1} \sin \phi_{1}-j A_{1} \cos \phi_{1}\right)} \\
& {\left[F T\left(U_{2}\right)\right]_{\omega_{n}}=\frac{1}{\omega_{n}}\left(A_{2} \sin \phi_{2}-j A_{2} \cos \phi_{2}\right)}
\end{aligned}
$$

which can be combined in the manner of equation (4) to give:

$$
\left[F T\left(U_{0}\right)\right]_{\omega_{n}}=\frac{1}{2 \omega_{n}}\left(\left(A_{1} \sin \phi_{1}+A_{2} \sin \phi_{2}\right)-j\left(A_{1} \cos \phi_{1}+A_{2} \cos \phi_{2}\right)\right)
$$

The real (denoted $\mathfrak{R}$ ) and imaginary (denoted $\mathfrak{J})$ parts of $\left[F T\left(U_{0}\right)\right]_{\omega_{n}}$ can therefore be written in terms of the real and imaginary parts of the original acceleration measurements:

$$
\begin{aligned}
& \Re\left[F T\left(U_{0}\right)\right]_{\omega_{n}}=\frac{1}{2 \omega_{n}}\left(\mathfrak{I}\left[F T\left(a_{1}\right)\right]_{\omega_{n}}+\mathfrak{I}\left[F T\left(a_{2}\right)\right]_{\omega_{n}}\right) \\
& \mathfrak{I}\left[F T\left(U_{0}\right)\right]_{\omega_{n}}=-\frac{1}{2 \omega_{n}}\left(\mathfrak{R}\left[F T\left(a_{1}\right)\right]_{\omega_{n}}+\mathfrak{R}\left[F T\left(a_{2}\right)\right]_{\omega_{n}}\right)
\end{aligned}
$$

Having acquired acceleration spectra and extracted real and imaginary parts, equations (12a\&b) are readily formulated in post-processing in, for example, standard numerical analysis software;

Microsoft Excel was used in this study.

\section{Correction for instrument only vibration - time delay adjustment}

An initial experiment in which the target motion was nominally zero but with significant instrument vibration ( $160 \times 10^{-3} \mathrm{~g}$ RMS broadband) showed excellent amplitude agreement between $U_{m}$ and $U_{0}$ in line with that shown previously in Figure 5b. Agreement between the phases of $U_{m}$ and $U_{0}$, however, showed an error that increased dramatically and linearly with frequency as shown in Figure 7 (dashed curve) and as previously seen in a comparison of LDV and accelerometer measurements [8]. This phase ramp is symptomatic of a time delay between the LDV and accelerometer channels. The phase delay is $\omega_{n} \tau$ where $\tau$ is the time delay between the channels and can effectively be found as the gradient of the plot of phase difference as a function of frequency. With the vibrating LDV selected as the reference device, the delays can be incorporated in the expressions for acceleration, i.e. the measured acceleration at time $t$ is the actual acceleration at time $\left(t-\tau_{1,2}\right)$. The accelerations are then re-written in the complex form as:

$$
\begin{aligned}
& {\left[a_{1}\right]_{\omega_{n}}=A_{1} \exp \left[j\left(\omega_{n}\left(t-\tau_{1}\right)+\phi_{1}\right)\right]} \\
& {\left[a_{2}\right]_{\omega_{n}}=A_{2} \exp \left[j\left(\omega_{n}\left(t-\tau_{2}\right)+\phi_{2}\right)\right]}
\end{aligned}
$$

[INSERT: Figure 7. Example phase difference between vibrating LDV measurement and correction measurement before and after time delay adjustment]

Following the same manipulations as above in equation ( $8 \mathrm{a} \& \mathrm{~b})$ to (11), the velocity $U_{0}$ can be written: 


$$
\begin{aligned}
{\left[F T\left(U_{0}\right)\right]_{\omega_{n}}=} & \frac{1}{2 \omega_{n}}\left(A_{1} \sin \left(\phi_{1}-\omega_{n} \tau_{1}\right)+A_{2} \sin \left(\phi_{2}-\omega_{n} \tau_{2}\right)\right) \\
& -j \frac{1}{2 \omega_{n}}\left(A_{1} \cos \left(\phi_{1}-\omega_{n} \tau_{1}\right)+A_{2} \cos \left(\phi_{2}-\omega_{n} \tau_{2}\right)\right)
\end{aligned}
$$

in which the effect of the time delays is clear when comparing to equation (11). It is useful to re-write in terms of real and imaginary parts as:

$$
\begin{aligned}
\mathfrak{R}\left[F T\left(U_{0}\right)\right]_{\omega_{n}}= & \frac{1}{2 \omega_{n}}\left(\cos \omega_{n} \tau_{1} \mathfrak{J}\left[F T\left(a_{1}\right)\right]_{\omega_{n}}+\cos \omega_{n} \tau_{2} \mathfrak{J}\left[F T\left(a_{2}\right)\right]_{\omega_{n}}\right) \\
& -\frac{1}{2 \omega_{n}}\left(\sin \omega_{n} \tau_{1} \mathfrak{R}\left[F T\left(a_{1}\right)\right]_{\omega_{n}}+\sin \omega_{n} \tau_{2} \mathfrak{R}\left[F T\left(a_{2}\right)\right]_{\omega_{n}}\right) \\
\mathfrak{I}\left[F T\left(U_{0}\right)\right]_{\omega_{n}}= & -\frac{1}{2 \omega_{n}}\left(\cos \omega_{n} \tau_{1} \mathfrak{R}\left[F T\left(a_{1}\right)\right]_{\omega_{n}}+\cos \omega_{n} \tau_{2} \mathfrak{R}\left[F T\left(a_{2}\right)\right]_{\omega_{n}}\right) \\
& -\frac{1}{2 \omega_{n}}\left(\sin \omega_{n} \tau_{1} \mathfrak{I}\left[F T\left(a_{1}\right)\right]_{\omega_{n}}+\sin \omega_{n} \tau_{2} \mathfrak{I}\left[F T\left(a_{2}\right)\right]_{\omega_{n}}\right)
\end{aligned}
$$

Figure 7 also shows the phase difference (solid curve), with mean value of zero, once the determined time delays (on the order of $0.65 \mathrm{~ms}$ for both $\tau_{1}$ and $\tau_{2}$ in this particular scenario) have been accounted for.

Figure 8a shows comparison between the original measured, $U_{m}$, and corrected, $U_{c o r r}$, velocities with respect to the 'true' target vibration. While, on initial inspection of these data, correction performance may seem to be limited, it should be noted that the amplitude error reduction, as shown in Figure 8b, is significant with an average level in excess of $33 \mathrm{~dB}$.

[INSERT: Figure 8. Comparison between original and corrected LDV measurements with respect to the 'true' target vibration (stationary target, instrument vibration at $160 \times 10^{-3} \mathrm{~g}$ RMS broadband); mean a) amplitudes and $b$ ) $d B$ reduction]

Correction for simultaneous instrument and target vibration - laboratory measurement

A second laboratory experiment was conducted using the same experimental arrangement as shown in Figure 6, although this time with both target ( $80 \times 10^{-3} \mathrm{~g}$ RMS broadband) and instrument vibration (160 $\times 10^{-3} \mathrm{~g}$ RMS broadband) applied. This scenario is intended to be representative of the kind of situation which might be experienced in a "real world" application. As expected, substantial differences are observed, as shown in Figure 9a, between the measurement from the vibrating LDV (dashed curve) and the 'true' measurement device (dotted curve). After correction, however, excellent agreement is found, also shown in Figure 9a, between the corrected measurement (solid curve) and the 'true' vibration. The reduction in correction performance which can be observed at the lower frequencies can be attributed to the reduced performance of the piezoelectric accelerometers at these lower frequencies; for improved low frequency performance, correction sensors with better low frequency performance would be necessary. As for the scenario with instrument vibration only, the average error reduction is significant at over $37 \mathrm{~dB}$ as can be seen from Figure 9b. Excellent 
agreement is also found between the phase of the corrected measurement and the 'true' measurement except for regions where genuine target vibrations are very low, as shown in Figure 9c. Note that, in these data, the 'true' measurement taken with the PDV100 has also been time-delay adjusted with respect to the OFV4000 instrument, in exactly the same way as for the accelerometers, based on a time-delay calculation conducted in advance from a measurement in which only the target was vibrating.

[INSERT: Figure 9. Comparison between vibrating LDV measurement before and after correction with 'true' target vibration (instrument vibration at $160 \times 10^{-3} \mathrm{~g}$ RMS broadband and target vibration at $80 \times 10^{-3} \mathrm{~g}$ RMS broadband); mean a) amplitudes, b) dB reduction and c) phase difference]

Correction for simultaneous instrument and target vibration - "real world" measurement

A final experiment was conducted during this study in order to demonstrate the effectiveness of the technique for a "real world" application. As shown in Figure 10, the LDV, fitted with the two correction accelerometers as before, was tripod mounted on a vehicle simulator "6-poster" platform which was programmed to undergo significant levels of six degree-of-freedom vibration. The target was the steering wheel on which a target reference accelerometer was mounted to provide the 'true' measurement. For these "field" measurements, time-delays for all three accelerometer channels were determined in advance in laboratory tests using a broadband (white noise) signal in the frequency range of interest. Time-delay adjustments all on the order of $0.55 \mathrm{~ms}$ were found in this case. As before, integration to velocity and incorporation of the necessary inter-channel time delays were achieved using equations (15a\&b).

[INSERT: Figure 10. Experimental arrangement for "real world" vehicle simulator vibration measurements]

Figure 11a clearly shows the substantial difference in amplitude between the original measurement from the vibrating LDV (dashed curve) and that measured by the reference accelerometer (dotted curve). Following correction (solid curve), the differences are significantly reduced; an average level reduction of in excess of $19 \mathrm{~dB}$ is found for the region of significant vibration from 2.5-30 Hz. As for the lab-based equivalent measurement, phase errors are small where the amplitude is high and larger elsewhere, as shown in Figure 11b. These data show clearly the potential for significant measurement error and the effectiveness of this important correction technique.

[INSERT: Figure 11. Comparison between vibrating LDV measurement before and after correction with 'true' measurement for "real world" application; mean a) amplitude and b) phase]

\section{Conclusions}

When the optical head of a LDV is itself subject to motion in the direction of the laser beam, the measurements made are as sensitive to this motion as they are to the target motion that it is intended 
to measure. This paper has confirmed this sensitivity experimentally and demonstrated the potential for erroneous measurements under such circumstances. It has also been shown that the erroneous measurement can be completely corrected by determination and subtraction of the instrument motion. This yields an absolute, rather than relative, vibration measurement enabling accurate "off-the-tripod" LDV measurements, e.g. when handheld or when fixed to a moving platform.

Given that an independent measurement of the instrument vibration velocity in the direction the laser beam at some point along the laser beam path is not practically achievable, a vector-based mathematical approach was used to define the requirements of the necessary correction measurements. Correction by a single accelerometer mounted on the instrument body with its sensitive axis aligned with the laser beam is not sufficient for accurate correction. Instead, a pair of sensors mounted with equal but opposite coordinates in the two directions orthogonal to the laser beam direction are required. Just two sensors deliver theoretically perfect correction, at any frequency, even for arbitrary six degree-of-freedom motion of the LDV optical head.

Measured signals were processed in the frequency domain, where integration of the accelerometer signals and correction of inter-channel time delays (circa $0.6 \mathrm{~ms}$ ) can be conveniently accomplished. In laboratory tests, error reductions in excess of $30 \mathrm{~dB}$ were achieved for measurements on stationary and vibrating targets. Phase errors were also low away from frequencies with low genuine motion; evaluating phase difference between signals at low levels is always practically challenging. Finally, a "real world" measurement from the steering wheel on a vibrating vehicle simulator platform was successfully undertaken with error reduction up to $19 \mathrm{~dB}$ and good phase agreement between corrected and 'true' measurements. Depending upon the specific nature of the target surface vibration of interest and that of the instrument itself the correction might, of course, be more or less effective at particular frequencies as can be seen in much of the data presented herein. While specific frequency ranges were selected for the sake of clarity of data presentation and validation of the proposed method, it is important to note that there is no restriction to the effective range in practice.

The work described in this paper is of fundamental importance to the vibration measurement community. For the first time, accurate measurements have been performed in the presence of significant levels of instrument vibration, effectively turning the LDV into an absolute, rather than relative, vibration measurement transducer. This eliminates the need for palliative anti-vibration mounting of the vibrometer and extends the range of LDV applications to include measurements from vibrating platforms such as the hand or vehicles on road and rail, in air or at sea. Such applications will be the topic of future research in this area as will extension to other LDV instrument variants. For example, scanning LDV measurements will be correctable in similar fashion, while for angular vibrations, including torsional vibrations, instruments have inherent insensitivity to translational vibrations through their parallel beam configuration but may require correction for angular vibration. 


\section{Acknowledgements}

The authors wish to acknowledge the support of the Control and Reliability Research Group within the Department of Aeronautical and Automotive Engineering at Loughborough University for support with the vehicle simulator facility.

\section{REFERENCES}

[1] Y. Li, P. Segers, J Dirckx \& R. Baets, On-chip laser Doppler vibrometer for arterial pulse wave velocity measurement, Biomed. Opt. Exp. 4(7) (2013) 1229-1235.

[2] G.M. Revel, P. Castellini, P. Chiariotti, E.P. Tomasini, F. Cenedese and A. Perazzolo, Laser vibrometry vibration measurements on vehicle cabins in running conditions: helicopter mockup application, Opt. Eng. 50(10) (2011) 101502-1-14.

[3] A. Dräbenstedt, X. Cao, U. Polom, F. Pätzold, T. Zeller, P. Hecker, V. Seyfried and C. Rembe, Mobile Seismic Exploration, to be published in Proc. $12^{\text {th }}$ Int. Conf. on Vib. Meas. by Las. and Noncont. Techs. (2016).

[4] P. Meldahl, E. Skomedal, P. Hanssen, A. Brendsdal, E. Vikhagen, R. Cleave, T. Bakke and K.J. Tjøm, Temprei - a robotized, contactless, multi-component seismic-receiver system, Proc. $77^{\text {th }}$ EAGE Conf. \& Exhib. (2015).

[5] H. Kim, Y. Lee, C. Kim, T.G. Chang and M.S. Kang. Laser Doppler vibrometer with body vibration compensation. Optical Engineering, 42(8) (2003) 2291-2295.

[6] L.A. Jiang, M.A. Albota, R.W. Haupt, J.G. Chen and R.M. Marino. Laser vibrometry from a moving ground vehicle. Applied optics, 50(15) (2011) 2263-2273.

[7] P. Martin and S.J Rothberg, Pseudo-vibration sensitivities for commercial laser vibrometers. Mech. Syst. Signal Process. 25(7) (2011) 2753-2765. DOI: 10.1016/j.ymssp.2011.02.009

[8] B.J. Halkon and S.J. Rothberg, Correction of laser Doppler vibrometry measurements affected by steering mirror vibration, Proc. OPTIMESS III (2016) 117-126. 


\section{Figure captions}

Figure 1. Experimental arrangement showing vibrating and fixed LDVs to demonstrate sensitivity to instrument vibration; a) schematic and b) physical set-up

Figure 2. Comparison between measurements from the vibrating LDV and from the fixed LDV on a (nominally) stationary target

Figure 3. For a nominally stationary target, comparison between measurements from the vibrating LDV and an accelerometer mounted on the vibrometer optical head body to one side of the beam emitter tube; instrument vibration $160 \times 10^{-3} \mathrm{~g}$ RMS broadband

Figure 4. Schematic showing required location of accelerometers to enable effective correction for instrument vibration in the direction of the laser beam

Figure 5. Comparison between LDV measurement (stationary target, instrument vibration at $160 \times 10^{-3} \mathrm{~g}$ RMS broadband) and alternative correction measurements: a) single accelerometer b) optimum combination of two accelerometers

Figure 6. Equipment arrangement for laboratory-based experimental investigation:

a) schematic and b) physical set-up

Figure 7. Example phase difference between vibrating LDV measurement and correction measurement before and after time delay adjustment

Figure 8. Comparison between original and corrected LDV measurements with respect to the 'true' target vibration (stationary target, instrument vibration at $160 \times 10^{-3} \mathrm{~g}$ RMS broadband); mean a) amplitudes and $b) \mathrm{dB}$ reduction

Figure 9. Comparison between vibrating LDV measurement before and after correction with 'true' target vibration (instrument vibration at $160 \times 10^{-3} \mathrm{~g}$ RMS broadband and target vibration at $80 \times 10^{-3}$ g RMS broadband); mean a) amplitudes, b) dB reduction and c) phase difference

Figure 10. Experimental arrangement for "real world" vehicle simulator vibration measurements Figure 11. Comparison between vibrating LDV measurement before and after correction with 'true' measurement for "real world" application; mean a) amplitude and b) phase 


\section{Figures}
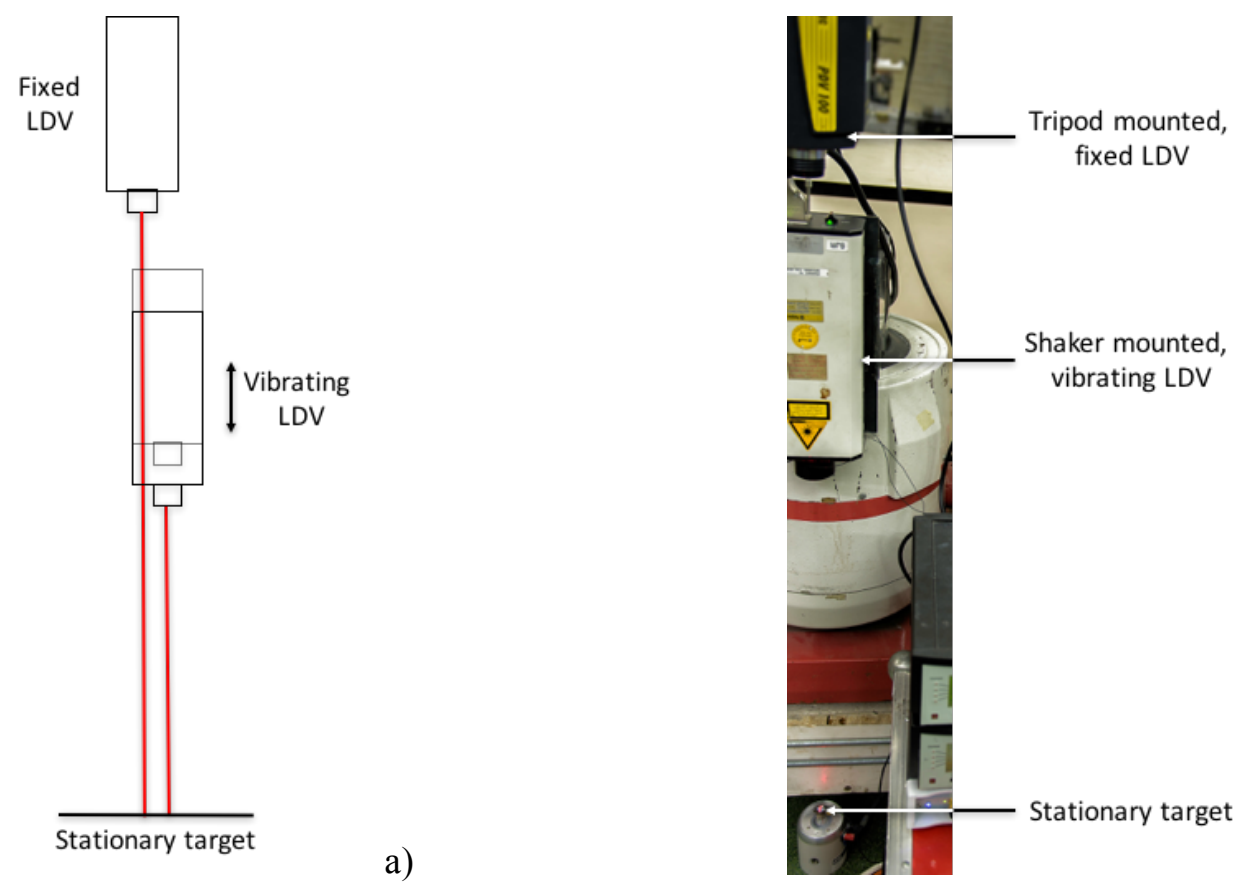

b)

Figure 1. Experimental arrangement showing vibrating and fixed LDVs to demonstrate sensitivity to instrument vibration; a) schematic and b) physical set-up

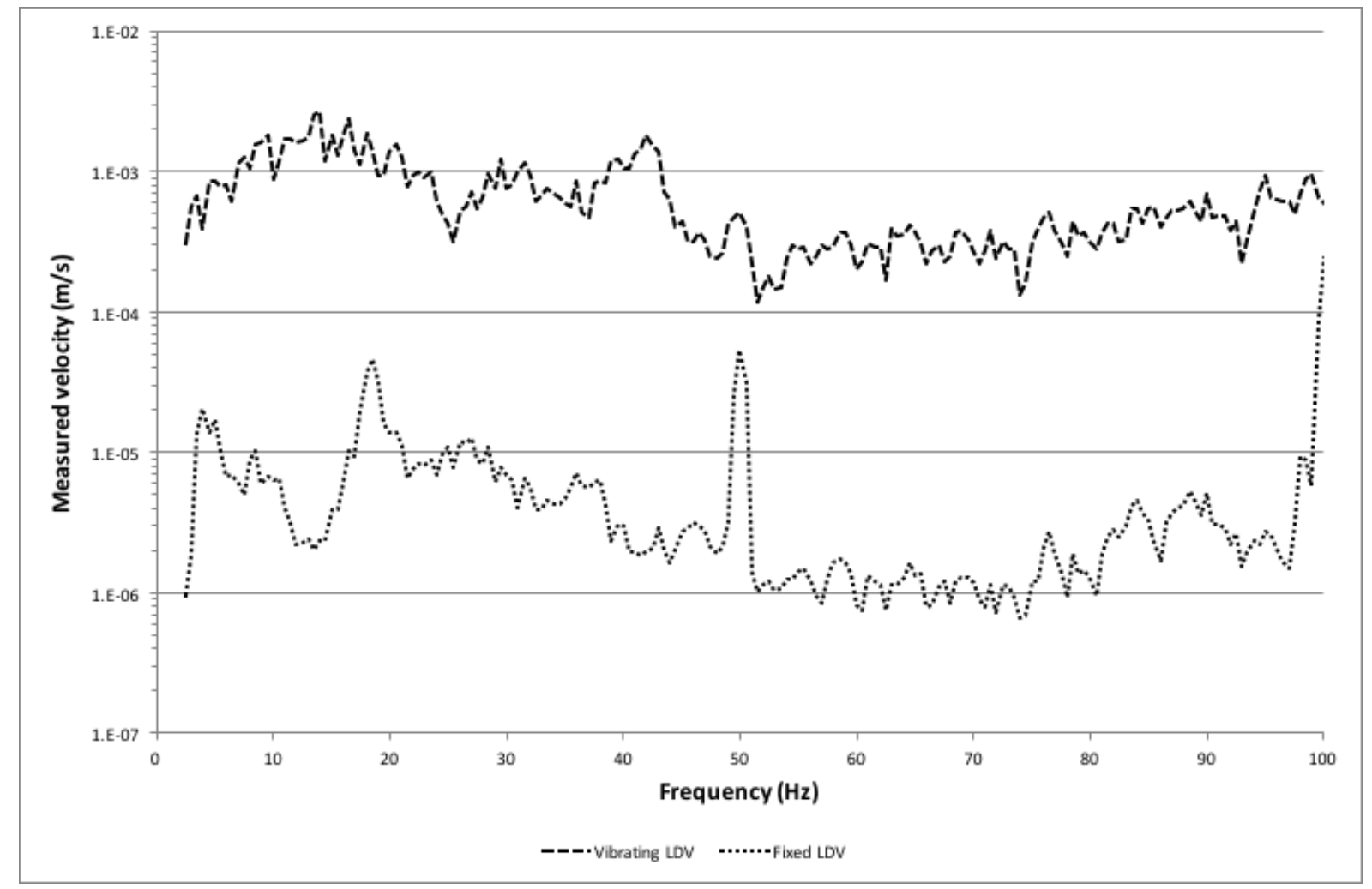

Figure 2. Comparison between measurements from the vibrating LDV and from the fixed LDV on a (nominally) stationary target 


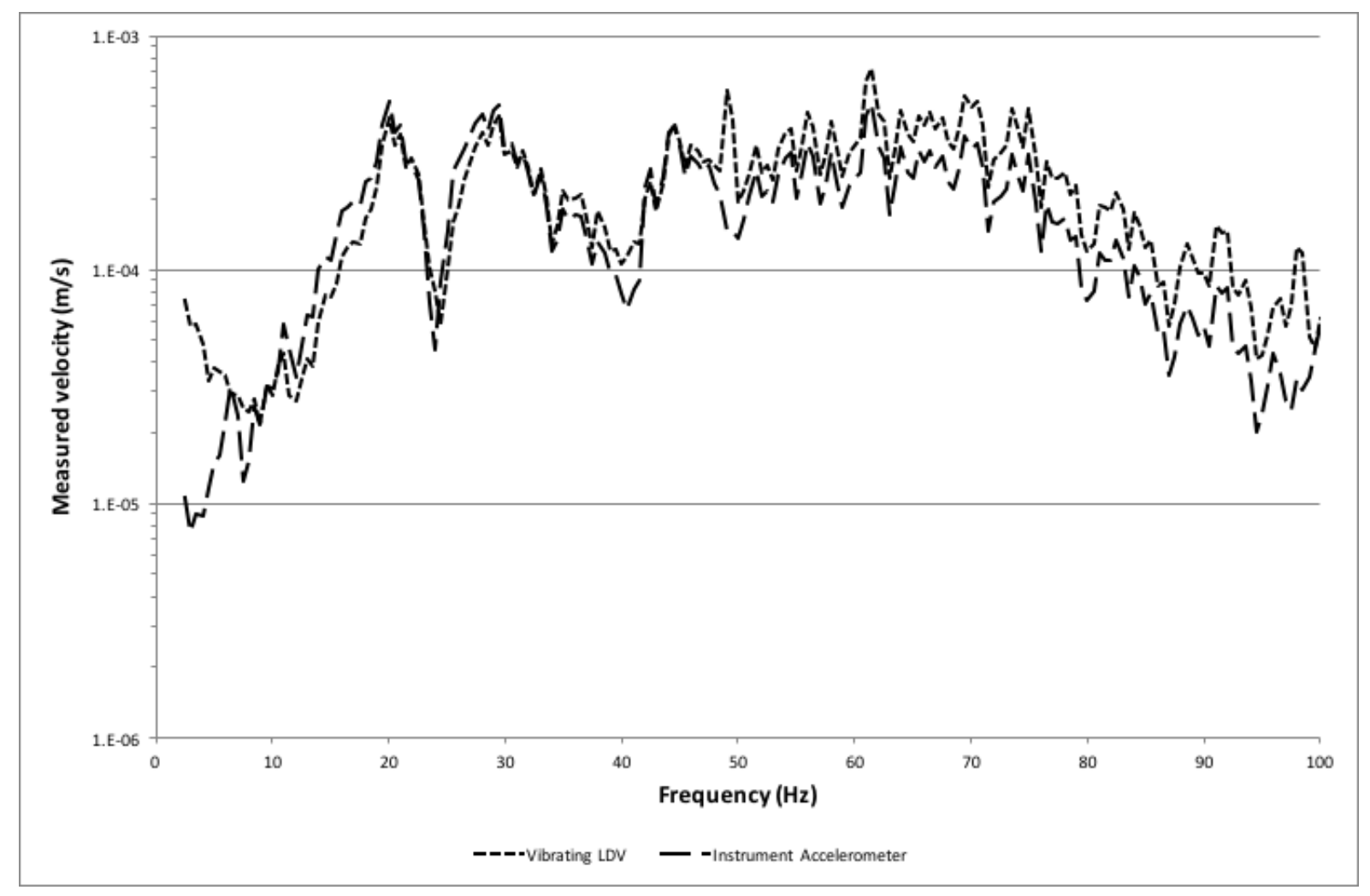

Figure 3. For a nominally stationary target, comparison between measurements from the vibrating LDV and an accelerometer mounted on the vibrometer optical head body to one side of the beam emitter tube; instrument vibration $160 \times 10^{-3} \mathrm{~g}$ RMS broadband

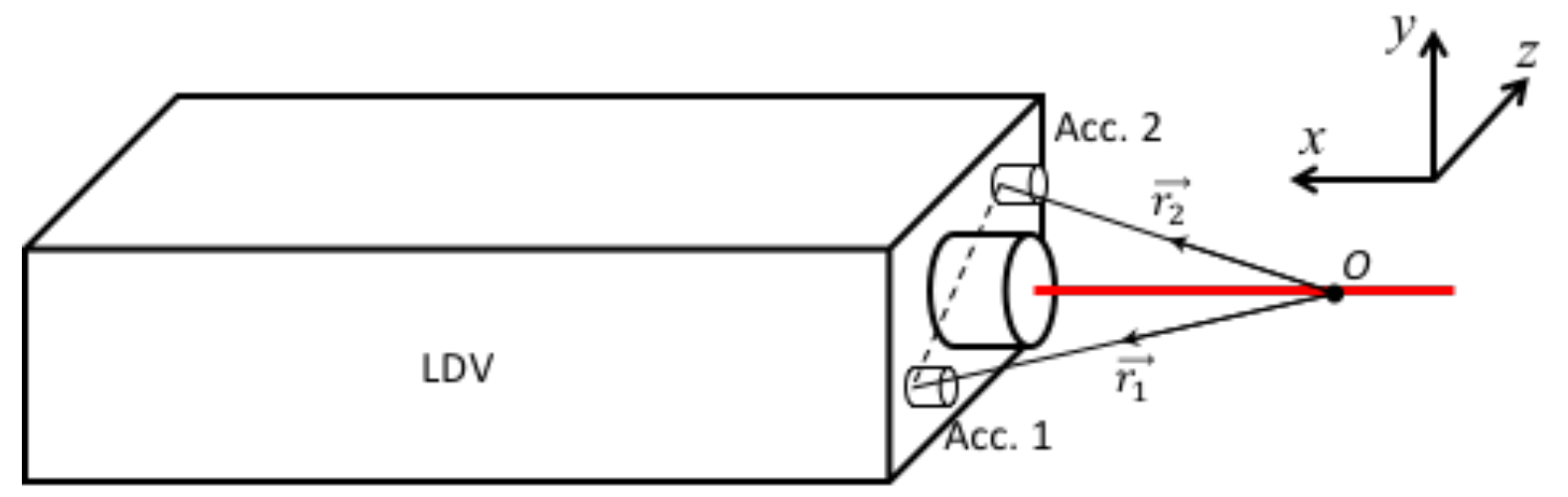

Figure 4. Schematic showing required location of accelerometers to enable effective correction for instrument vibration in the direction of the laser beam 

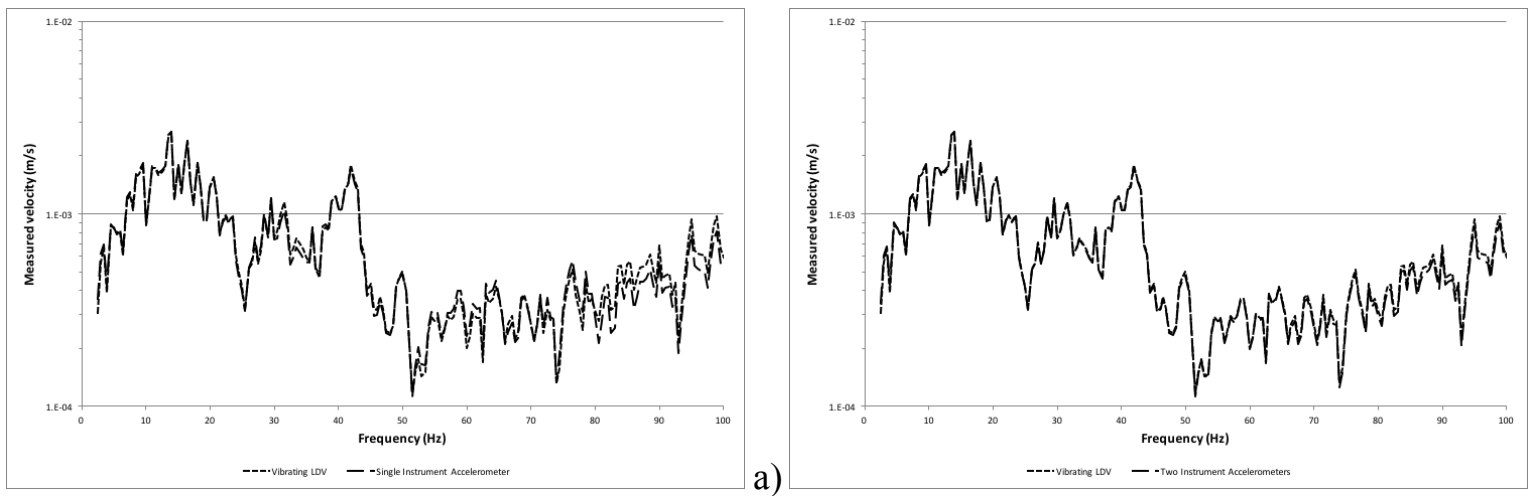

a)

b)

Figure 5. Comparison between LDV measurement (stationary target, instrument vibration at $160 \times 10^{-3} \mathrm{~g}$ RMS broadband) and alternative correction measurements: a) single accelerometer b) optimum combination of two accelerometers

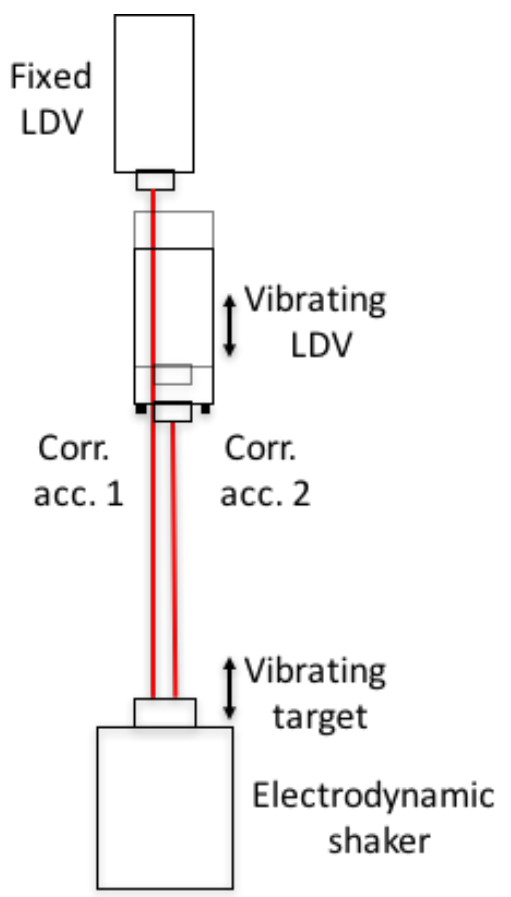

a)

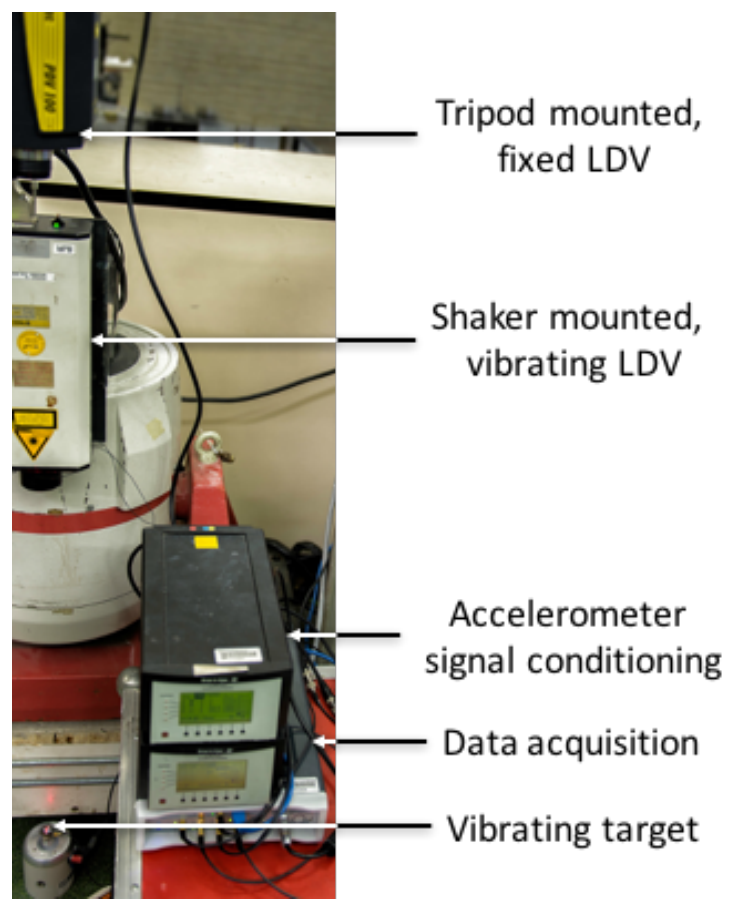

Figure 6. Equipment arrangement for laboratory-based experimental investigation:

a) schematic and b) physical set-up 


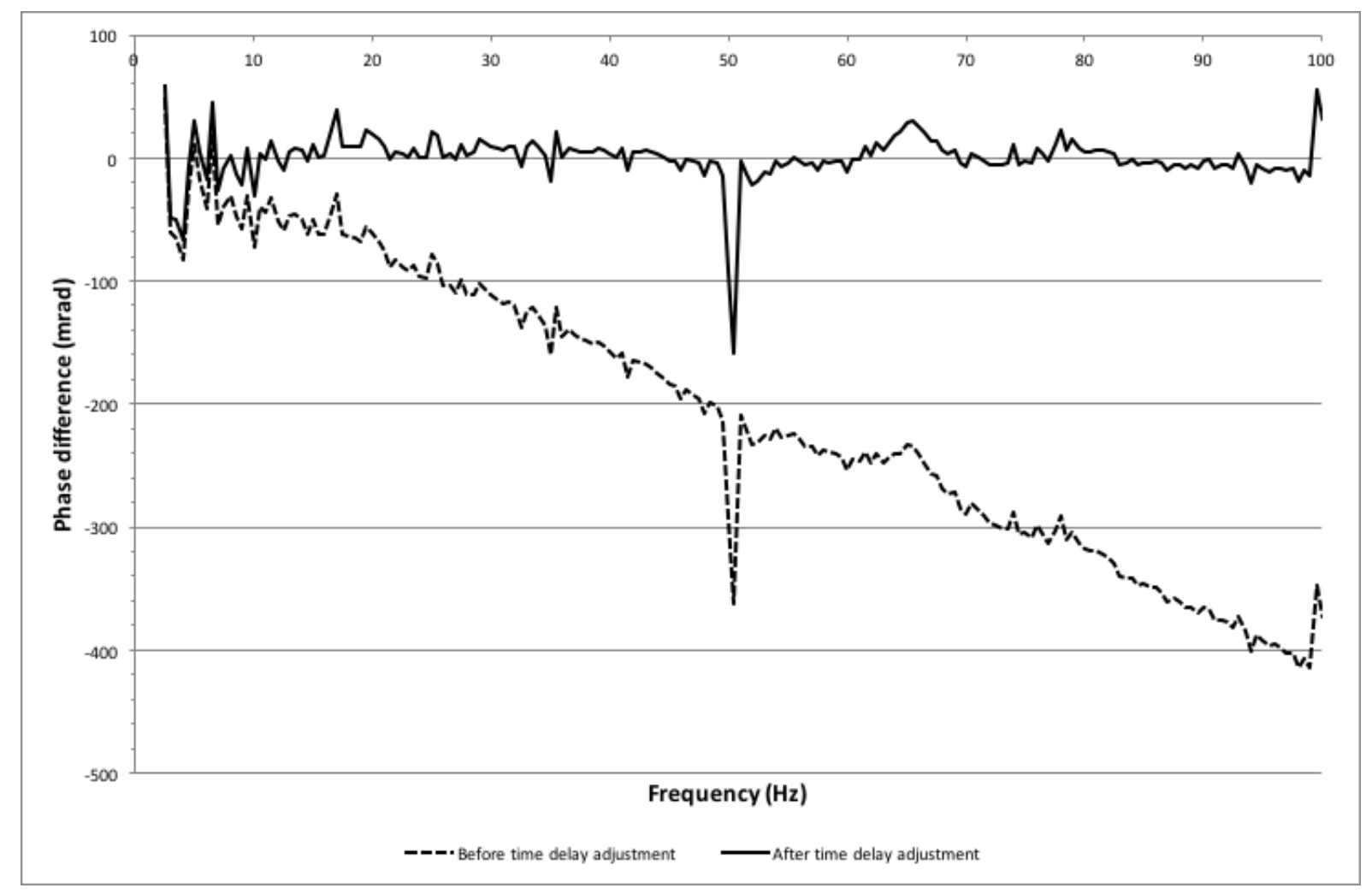

Figure 7. Example phase difference between vibrating LDV measurement and correction measurement before and after time delay adjustment
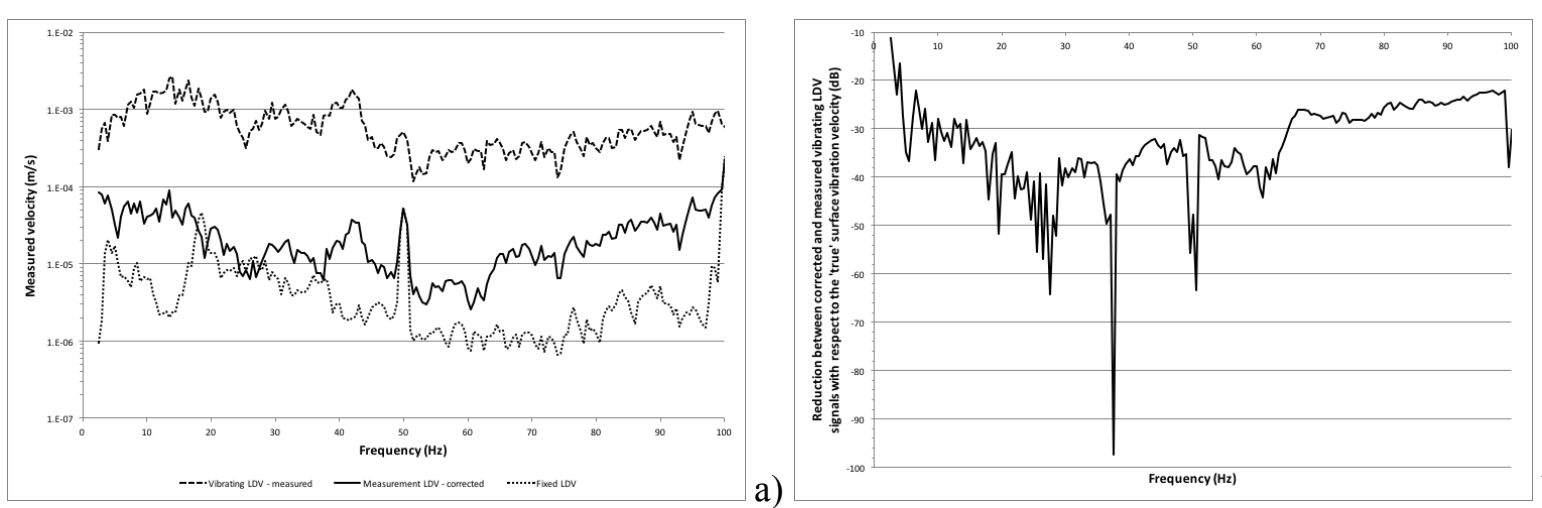

Figure 8. Comparison between original and corrected LDV measurements with respect to the 'true' target vibration (stationary target, instrument vibration at $160 \times 10^{-3} \mathrm{~g}$ RMS broadband); mean a) amplitudes and b) dB reduction 

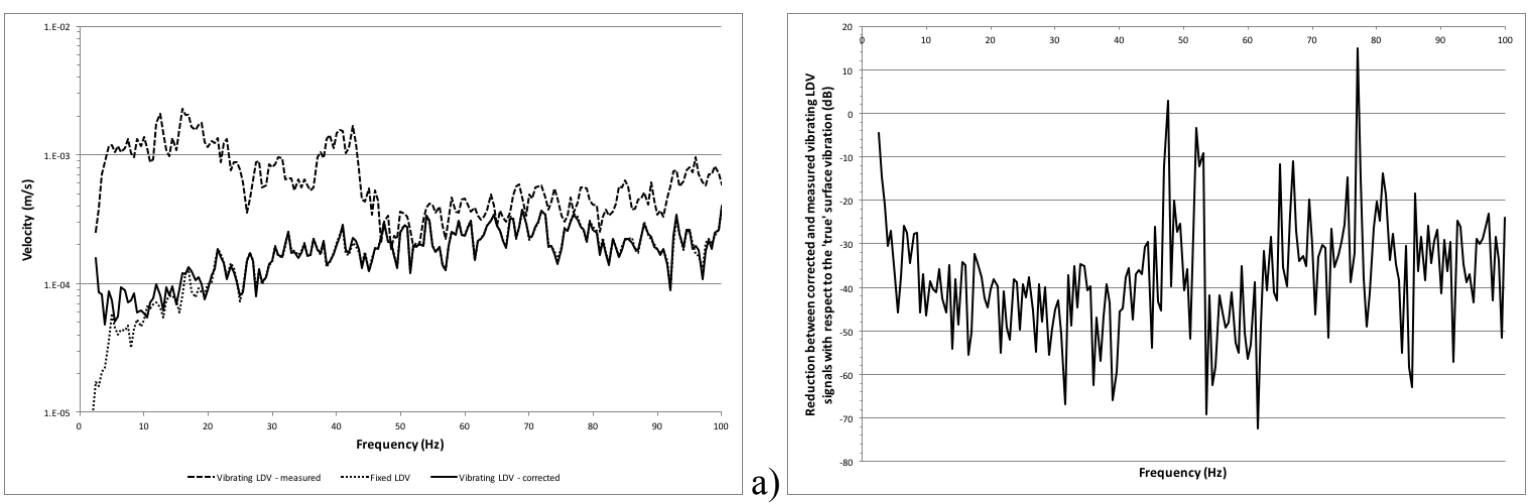

a)

b)

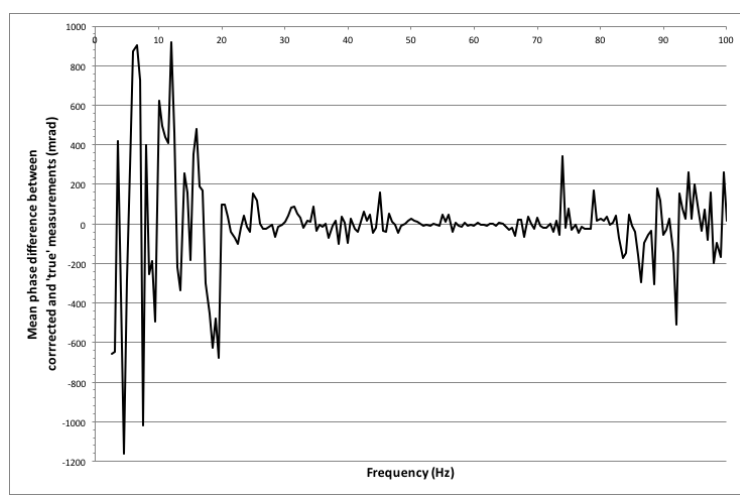

c)

Figure 9. Comparison between vibrating LDV measurement before and after correction with 'true' target vibration (instrument vibration at $160 \times 10^{-3} \mathrm{~g}$ RMS broadband and target vibration at $80 \times 10^{-3}$ g RMS broadband); mean a) amplitudes, b) dB reduction and c) phase difference 


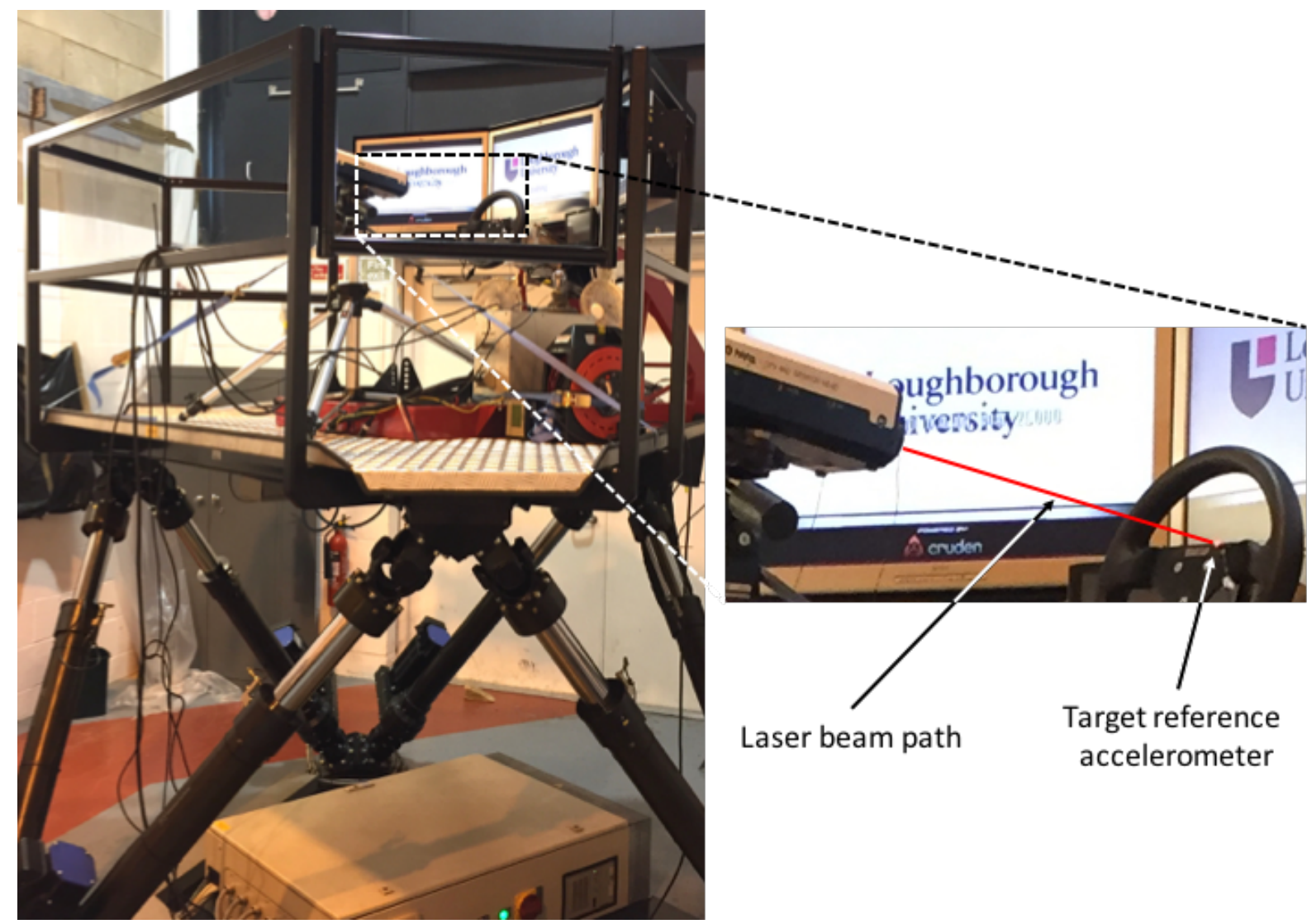

Figure 10. Experimental arrangement for "real world" vehicle simulator vibration measurements
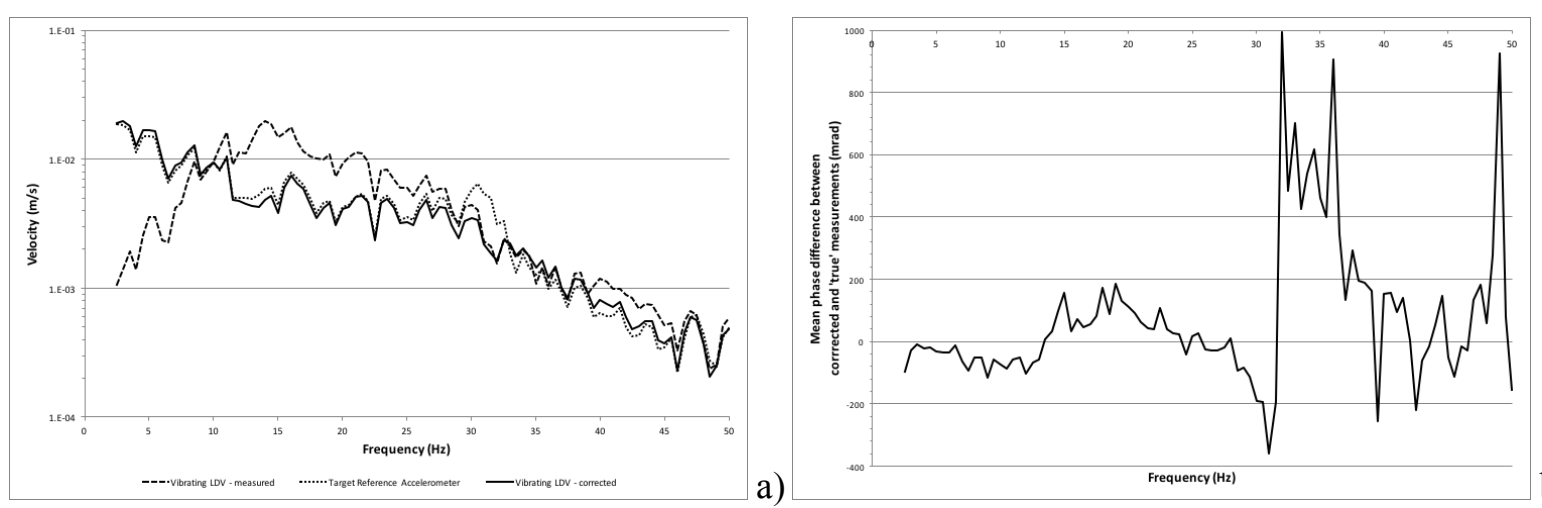

b)

Figure 11. Comparison between vibrating LDV measurement before and after correction with 'true' measurement for "real world" application; mean a) amplitude and b) phase 